\title{
Modeling X/ $/$-ray Spectra of Galactic Black Holes and Seyferts
}

\author{
Juri Poutanen \\ Stockholm Observatory, SE-13336 Saltsjöbaden, Sweden
}

\begin{abstract}
Recent high quality broad-band X-ray and gamma-ray observations of Galactic black holes by ASCA, Ginga, RXTE, and CGRO show that the spectra cannot be described by simple thermal Comptonization models (even including a fully relativistic treatment). Multi-component models accounting for Comptonization by thermal and non-thermal electrons, Compton reflection of $\mathrm{X} / \gamma$-rays from cold matter, and emission of the optically thick accretion disk are required. In this review, I discuss recent advances in the spectral modeling of Galactic black holes and Seyferts and the codes available for such an analysis.
\end{abstract}

\section{SPECTRAL MODELS}

Already at the time of the discoveries in the early 1970s of the first X-rays sources it was realized that the most efficient process to produce large $\mathrm{X}$-ray $/ \gamma$ ray ( $\mathrm{X} \gamma$ hereafter) luminosities is Comptonization of soft seed photons by a hot rarefied electron gas (e.g., [1]). Detailed calculations of thermal Comptonization spectra were carried out by Monte-Carlo [2-4] and analytical methods [1,5-7]. Analytical formulae describing spectra emerging from an optically thick cloud of nonrelativistic electrons have been derived by solving the Kompaneets equation [8] in a "double" diffusion approximation (in optical depth and frequency) [7] and have been used extensively during last twenty years to fit the $\mathrm{X} \gamma$ data. Only recently the data has become good enough to require a more accurate theoretical description of Comptonized spectra. The usefulness of generalizations of the analytical formulae to a mildly-relativistic gas and an optically thin media [9] is disputable [10] and not trivial, since both electron and photon energies can be of order $\sim m_{e} c^{2}$. No analytical formulae exist for Comptonized spectra for non-Maxwellian electron distribution (except for power-law distributions of electrons in the optically thin limit). Monte-Carlo simulations unfortunately, are still too slow to be implemented into the standard software for the $\mathrm{X} \gamma$ data analysis (e.g. XSPEC), but on the other hand give the possibility to consider arbitrary geometries.

All this makes attractive the use of numerical methods capable in solving relativistic kinetic equations for Compton scattering on electrons of arbitrary energy 
distribution without any restrictions on photon and electron energies in a reasonably short computer time. Such codes have become available recently [11,12]. Some codes (XSPEC versions NTEEA [13] and EQPAIR [14]) follow not only the photon, but also the electron distribution. They also allow, in a self-consistent manner, the determination of the electron temperature, optical depth, and such physical quantities as, e.g., the heating rate of thermal particles, the efficiency of acceleration of nonthermal particles, directly from observations.

An important ingredient of spectral modeling is an accurate treatment of the reflection of X $\gamma$-rays from the cold material ("Compton reflection") in the vicinity of the compact object (accretion disk) [15]. The reflected spectrum depends on a number of factors: ionization state of the material, element abundances, angular distribution of the incident photons, and, of course, their spectral distribution (and polarization). A number of models simulating Compton reflection is implemented into XSPEC. The most developed model is probably PEXRIV [16]. It incorporates exact Green's functions accounting for the angular dependence of the outgoing (observed) reflected spectrum and different ionization states of the reflecting medium, while being self-consistent regarding the ionization balance only up to a reflector temperature of order $0.1 \mathrm{keV}$ [17]. If the incident spectrum is anisotropic as expected, e.g., in the case of magnetic flares on the surface of accretion disks [18-21], the reflected spectrum should be computed using Green's functions that account for angular dependence of both incident and reflected radiation [22].

Rotation of the accretion disk can also be an important factor causing measurable distortion of the reflected spectrum. An exact solution for a Kerr black hole is far from being simple and is not yet available in XSPEC. A useful approximation, the convolution of the Compton reflection from the static matter with a relativistic disk line profile [23], has already been used to interpret smeared iron edges in some Galactic black holes (GBH) $[24,25]$.

\section{SEYFERTS AND THE HARD STATE OF GBH}

Historically, the thermal Comptonization model was used to fit the data from GBH (see, e.g., $[7,26,27]$ ). While the spectra of Seyfert galaxies were explained in terms of the nonthermal pair-reflection model [28], which well reproduced a quite small spread in the observed X-ray spectral indices [29]. The fact that the X-ray spectra of GBH in their hard state are quite similar to the spectra of Seyferts was realized quite early [30], while only recently they were shown to be similar also up to the soft $\gamma$-rays [31]. Probably, the only difference is that amount of Compton reflection is generally higher in Seyferts than in GBH [32-34]. Both GBH and Seyferts show a cutoff in their spectra at $\sim 100 \mathrm{keV}$, thus making thermal models more likely. The best available data yield a Thomson optical depth, $\tau \approx 1$, and a temperature, $k T_{e} \approx 50-100 \mathrm{keV}$, of the Comptonizing cloud.

Evidence for deviations from the (intrinsic) pure thermal Comptonization spectrum is rather weak in the case of Seyferts. None of them has been detected above 


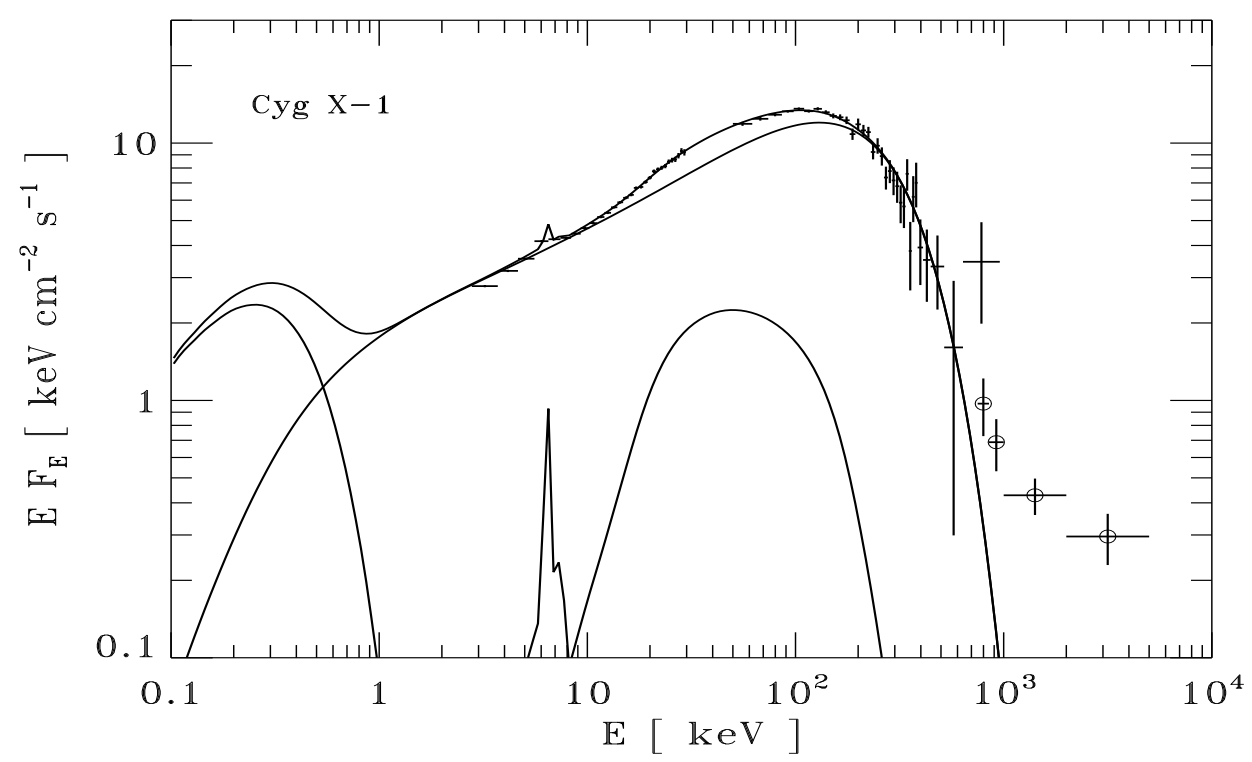

FIGURE 1. The hard state of Cygnus X-1. Simultaneous data from Ginga, OSSE and COMPTEL in June 1991. High energy excess is clearly visible in the COMPTEL data at $E \gtrsim 1$ $\mathrm{MeV}$. Different components correspond to the soft seed radiation from the accretion disk (or cold clouds mixed with the hot plasma), thermal Comptonized spectrum, and its Compton reflection (together with the fluorescent iron line) from a weakly ionized cold matter.

$\sim 500 \mathrm{keV}$ with modern instruments [35,36]. The fraction of power that can be channeled to the nonthermal particles (nonthermal efficiency) is $\lesssim 10$ per cent in the case of NGC 4151, the brightest Seyfert galaxy in X $\gamma$-rays [10]. On the other hand, observations by BATSE and COMPTEL revealed the presence of a high energy excess at $\gtrsim 500 \mathrm{keV}$ in two GBH, Cygnus X-1 and GRO J0422+32 [37-39] (see Fig. 1), that requires $\sim 10$ per cent nonthermal efficiency [40,41]. Electrons have an almost Maxwellian distribution with a weak power-law tail. Deviations from a Maxwellian distribution could also explain why a combination of two thermal Comptonization spectra was required to fit the data of Cyg X-1 [34], while a single component corresponding to Comptonization by a hybrid plasma [11] provides a very good fit to the data from $1 \mathrm{keV}$ up to $4 \mathrm{MeV}$ (see [41] and Fig. 4).

What is the geometrical arrangement of the hot and the cold matter? In a simple slab-corona model $[42,43]$, hot corona covers the whole cold disk. When most of the energy is dissipated in the hot phase, soft seed photons for Comptonization are produced only by reprocessing $\mathrm{X} \gamma$ radiation and the resulting spectrum is the hardest possible. However, it was recently shown that even these spectra are too steep to be consistent with observations of GBH and Seyferts [19,44-46]. In principle, the spectra from the slab-corona can be rather flat in the standard $\mathrm{X}$-ray region due to the anisotropy effects $[12,43]$ which become important for high electron temperature, but then the high energy cutoffs are not compatible with the data. Additional soft photons (besides the reprocessed ones), that could be 
generated in the cold disk, worsen the discrepancy.

Localized active regions (magnetic flares) on the surface of the accretion disk $[18,47]$ have less soft photons returning to the region than the hot slab has. Thus, being more photon starved, they produce harder spectra in consistence with observation. Due to the anisotropy of the radiation scattered once in the corona, the spectrum should have a break (anisotropy break, see [19-21]), which has never been observed. In the case of Seyferts, which can have smaller temperature of the seed photons, this break can be hidden below $\sim 1 \mathrm{keV}$ making its detection impossible due to interstellar absorption. The amount of Compton reflection in GBH is much smaller than expected from flares atop of an extended cold disk making this model questionable, at least for GBH (however, see [48]).

A hot inner disk and a cold outer disk model (e.g. [1,49]) would be compatible with both the observed spectral indices and the amount of Compton reflection. If the cold disk provides soft photons for cooling then, in the case of Cyg X-1 and GX339-4, its inner edge should penetrate into the hot region $[44,46]$ in order to provide enough reprocessed soft photons. The data are also compatible with a model where small dense cold clouds mixed with the hot media [50-53] reprocess $\mathrm{X} \gamma$ radiation and provide seed soft photons [46]. In any case, the outer disk should be flared in order to give rise to the observed amount of Compton reflection. As was already mentioned, Seyferts generally have a larger observed amplitude of Compton reflection than GBH. This can be related either to the different structure of the inner accretion disk, or just to the fact that a large amount of cool material (the molecular torus) $[54,55]$ surrounds the accretion disk in Seyfert galaxies providing additional reflection. Concluding, these models can unify the geometry of GBH and Seyferts' accretion disks.

It is quite difficult to quantify spectral variability even of the brightest GBH in the soft $\gamma$-rays, since the minimum integration time (with OSSE) needed to get a spectrum with a reasonable statistic at $\gtrsim 100 \mathrm{keV}$ is about one hour. In the hard state, there are evidences that Cyg X-1 shows an increase of the cutoff energy when the luminosity in the hard X-rays drops, while the spectrum in the standard X-ray band, 2-20 keV, hardly changes [34]. Changes in the optical depth, $\tau$, and electron temperature compensate each other in a way to keep the Kompaneets $y$-parameter constant. Since the temperature increases when the luminosity decreases, the spectra for different luminosities cross each other at about $\sim 500 \mathrm{keV}$. Such a behavior implies a constant ratio of the seed soft luminosity to the heating rate of the hot cloud. This is possible if the transition radius between hot and cold disks does not change much in the case the seed photons are internally produced in the outer cold disk. Alternatively, the seed photons could be provided mostly by reprocessing of the $\mathrm{X} / \gamma$-rays.

GBH are highly variable on all possible time scales (see, e.g., [56]). Of course, a successful model must explain both spectral and temporal properties. The size of the $\mathrm{X} \gamma$ source inferred from the time lags between hard and soft photons $[57,58]$ is a factor of $\sim 100$ larger than the size inferred from spectral modeling, assuming that the lags are related to the scattering time. One possible solution is to propose 


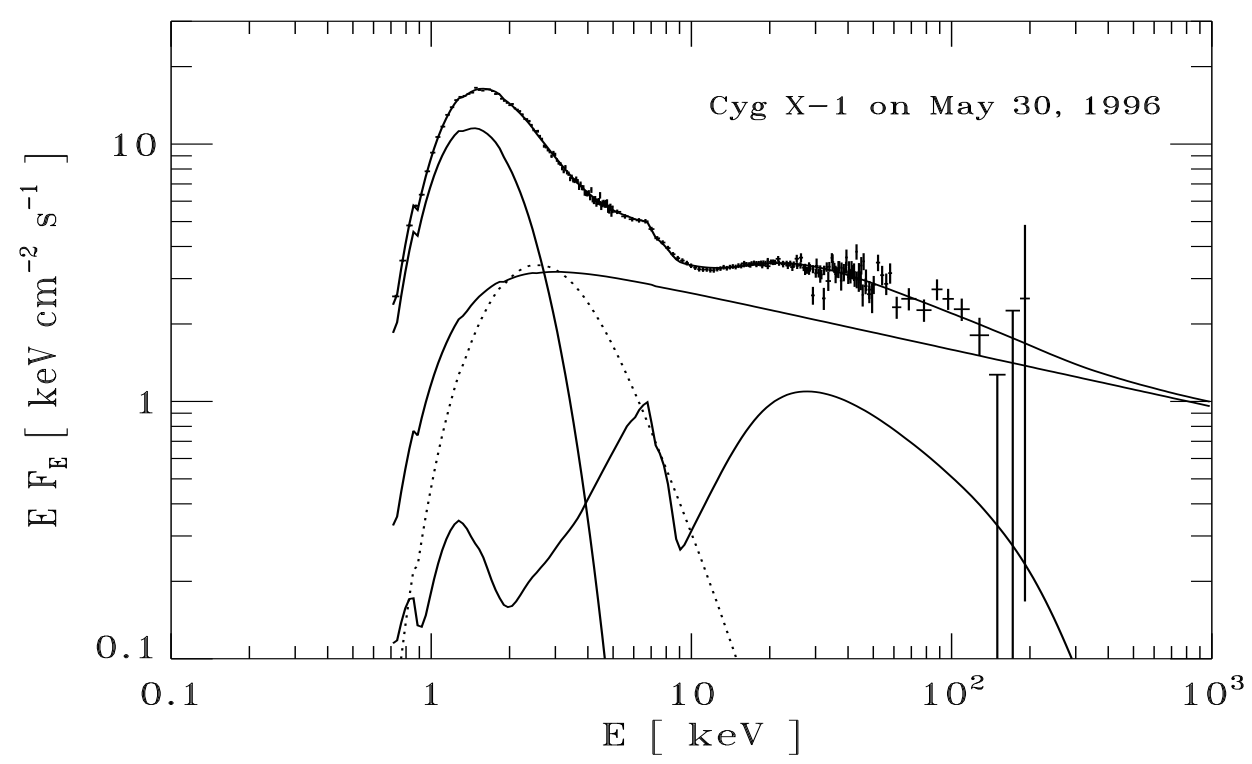

FIGURE 2. The soft state of Cygnus X-1. Simultaneous observations by $A S C A$ and $R X T E$ on May 30, 1996 [61]. The overall spectrum consists of disk emission, a non-thermal power-law, Compton reflection from an ionized disk, and an additional (thermal Comptonization) component peaking at $\sim 3 \mathrm{keV}$.

a large ( $\sim 10^{4}$ gravitational radii) size of the Comptonizing cloud [59,60], but it is difficult to imagine any mechanism that could heat the plasma up to 50-100 keV and dissipate most of the energy at such large distances from the black hole. On the other hand, it is possible that the time lags are related not to the scattering time, but, e.g., to the life-time of the magnetic flares that may be responsible for the energy dissipation.

\section{THE SOFT STATE OF GBH}

Unlike in the hard state, most of the luminosity in the soft state is carried by a blackbody-like component with a characteristic temperature, $k T_{\mathrm{bb}} \approx 0.3-1 \mathrm{keV}$. Figure 2 gives an example of the soft state spectrum of Cyg X-1 observed by $A S C A$ and $R X T E$ in 1996. A soft component (at $\lesssim 5 \mathrm{keV}$ ) cannot be represented by a black body, or by a multicolor disk spectrum, or by a modified black body. It is clear that at least two component are required to fit it (e.g., a black body and a powerlaw, or two black bodies [24,62]). The soft black body probably originates in the accretion disk. The nature of the additional component peaking at $\sim 3 \mathrm{keV}$ is not so clear. Gierliński et al. [24] interpreted it as due to the thermal Comptonization of a disk black body in a plasma with $k T_{e} \approx 5 \mathrm{keV}$ and $\tau \approx 3$.

The spectra of GBH in the hard X-rays/soft $\gamma$-rays can be well represented by a power-law without an observable break at least until $\sim m_{e} c^{2}$ [63-66]. COMPTEL has detected Cyg X-1 and GRO J1655-40 at energies up to $\sim 10 \mathrm{MeV}$ [67] and 
it seems that the power-law at $\mathrm{MeV}$ energies is just a continuation of the hard $\mathrm{X}$-ray power-law. If true, these observations would rule out the interpretation of the power-law as being due to bulk motion Comptonization in a converging flow $[68,69]$. This power-law suppose to have a break at $\lesssim m_{e} c^{2}$. Although the signatures of Compton reflection are also observed in this state (e.g., [70]), its magnitude, $R \equiv \Omega / 2 \pi$, is much more difficult to determine since it depends on the assumed run of ionization with radius and the detailed modeling of the continuum which is very curved around the iron edge. In case of $\mathrm{Cyg} \mathrm{X}-1, R \approx 0.6-0.8$ [34], and matter providing Compton reflection appears to be strongly ionized and the iron edge is smeared by relativistic effects close to the black hole.

The power-law can be produced by Comptonization of soft photons from the accretion disk by an optically thin nonthermal (probably, $e^{ \pm}$pair dominated) corona which covers much of the disk $[41,71,72]$. In terms of the hybrid thermal/nonthermal model of Coppi et al. [11,14], the nonthermal efficiency in the soft state is much higher than in the hard state, while the soft luminosity (from the accretion disk) exceeds both the thermal and nonthermal energy injection to the electrons [41]. The self-consistent electron distribution can be represented by a thermal distribution with a lower temperature (relative to the hard state) of $\sim 30$ $\mathrm{keV}$ and a significant power-law tail. The resulting photon spectrum is a black body from the cold accretion disk with a "soft excess" due to Comptonization by the thermal population of electrons (pairs), and a high energy power-law (Comptonization by non-thermal electrons) extending up to $\sim k T_{b b} \gamma_{\max }^{2}$ (here $\gamma_{\max }$ is the maximum electron energy).

\section{SPECTRAL TRANSITIONS IN CYG X-1}

What is really happening when a source changes from the hard to the soft state? In the case of $\mathrm{Cyg} \mathrm{X}-1$, when the $\mathrm{X} \gamma$ luminosity $(1 \mathrm{keV}-1 \mathrm{MeV})$ drops below

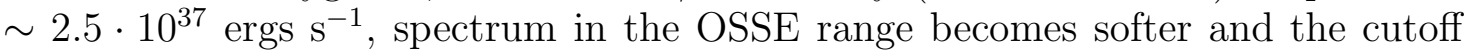
energy increases (see [66,73] and Fig. 3). The hard state OSSE spectra can be well described by a thermal bremsstrahlung spectrum even this model is clearly unphysical [1], and the bremsstrahlung temperature, $T_{b r}$, has no relation whatsoever to the physical electron temperature. When the source is in the hard state, $T_{b r}$ does not vary much $[66,74]$. During the transition, best fitted $T_{b r}$ decreases, but it is difficult to interpret this behavior, since thermal bremsstrahlung gives very poor fit to the data in the soft state, while a simple power-law with an exponential cutoff does a good job giving a superior fit.

In the hard state, the inner radius of the cold disk is far away from the black hole (at $\sim 30-50 G M / c^{2}$ ). Almost all the energy is dissipated thermally in the hot central cloud/corona. The $\mathrm{X} / \gamma$-ray source is photon-starved, since the luminosity of the outer cooler part of the accretion flow is small and the covering factor of the hot corona is quite small too. The spectrum is hard. The nonthermal energy injection rate is small as compared to the thermal dissipation rate. In the soft state, the 


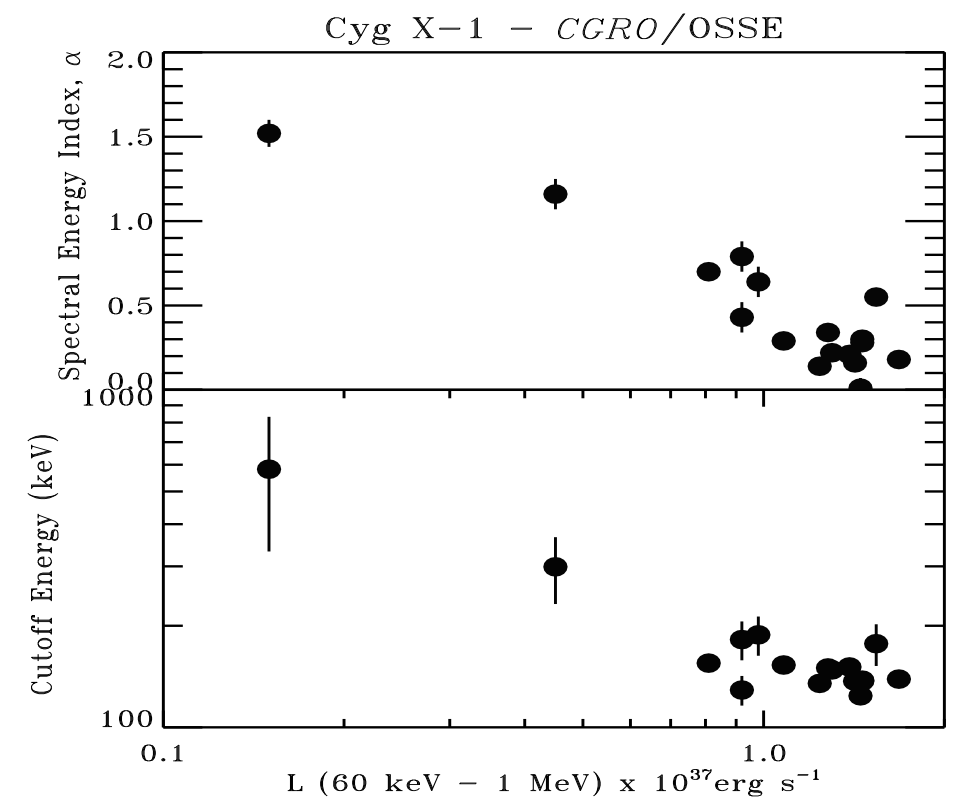

FIGURE 3. Dependence of the energy spectral index, $\alpha$, and cutoff energy, $E_{c}$, of the best fit model $f(E) \propto E^{-\alpha} \exp \left(-E / E_{c}\right)$ on the hard X-ray/soft $\gamma$-ray luminosity. Data are adapted from Table 3 of Phlips et al. [66].

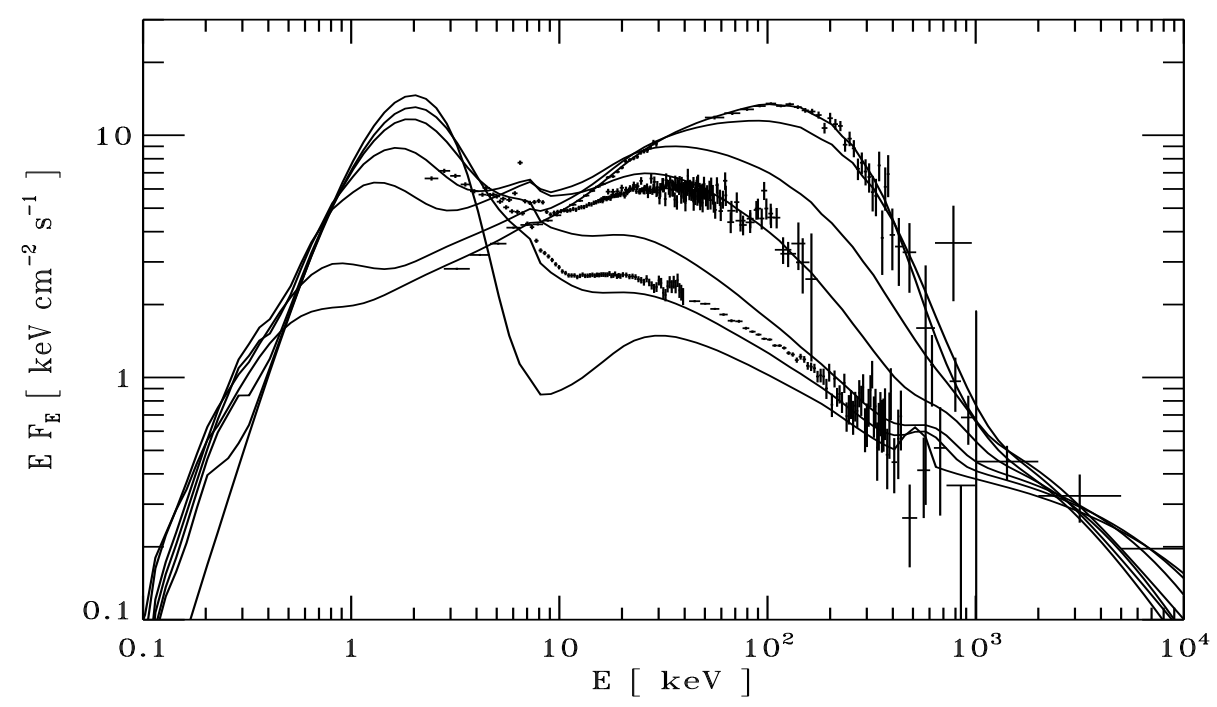

FIGURE 4. Spectral states of $\mathrm{Cyg} X-1$ and the state transition as predicted by the hybrid pair model. The sum of cold disk luminosity and the thermal dissipation rate in the hot disk remain constant. The nonthermal injection rate also does not change during the whole transition. Adapted from $[41,61]$. 
optically thick cool disk moves inwards and receives a major part of the dissipated energy $[44,75,76]$. The thermal energy dissipation rate in the corona is now a factor of $\sim 10$ lower than the soft luminosity (from the accretion disk) entering the corona. The rate of energy injection to the nonthermal electrons can stay constant during the transition. The sum of the soft luminosity from the cold disk and the thermal dissipation rate in the hot disk (corona) also remain approximately constant. Thus, the whole transition is consistent with just a redistribution of the energy input between the cold outer disk and the hot inner disk, with a constant nonthermal energy injection to the corona (see Fig. 4).

\section{SUMMARY}

During last twenty years, thermal Comptonization (nonrelativistic) models were used extensively to describe the data from accreting black holes. Only recently, the observational data became good enough to show that these models are only a zeroth order approximation and should be revised significantly. We learned that analytical nonrelativistic models do not describe the data in the hard X-rays. There are growing evidences that the energy distribution of electrons, responsible for Comptonizing soft photons, can deviate notably from a Maxwellian. Numerical methods have appeared that use an exact (fully relativistic) redistribution function for Compton scattering and account for accurate radiative transfer and the geometry of the Comptonizing cloud. These methods provide the emission spectrum from such plasma as well as a self-consistent electron (-positron) distribution.

Compton reflection was shown to modify the spectrum significantly and smearing of this component due to relativistic effects was observed. However, there are still no models for Compton reflection from a rotating accretion disk around a rotating black hole that would be implemented into the standard data analysis packages.

Spectral models considered here are, of course, oversimplified, since most of them do not consider mechanisms of energy dissipation. On the other hand, even the best developed accretion disk models still lack important ingredients (e.g. nonthermal power injection) that would allow them to describe broad-band data from the accreting black holes. We still lack models that can explain spectral and temporal properties together.

Most importantly, we need more broad-band simultaneous data to make firm conclusions about the physics of the accretion disks around black holes.

\section{ACKNOWLEDGMENTS}

I would like to thank the Swedish Natural Science Research Council and the Anna-Greta and Holger Crafoord's Fund for financial support. I am grateful to Paolo Coppi, Andrzej Zdziarski, Marek Gierliński, and Roland Svensson for valuable discussions and for providing me with some of the data used in this review. I thank Roland Svensson and Felix Ryde for comments on the manuscript. 


\section{REFERENCES}

1. Shapiro, S. L., Lightman, A. P., \& Eardley, D. N. 1976, ApJ, 204, 187

2. Gorecki, A. \& Wilczewski, W. 1984, Acta Astronomica, 34, 141

3. Pozdnyakov, L. A., Sobol, I. M., \& Sunyaev, R. A. 1983, in Sov. Sci. Rev. E Astrophys. Space Phys., edited by R. A. Sunyaev, vol. 2, 189

4. Stern, B. E., Begelman, M. C., Sikora, M., \& Svensson, R. 1995, MNRAS, 272, 291

5. Illarionov, A. F. \& Sunyaev, R. A. 1972, AZh, 49, 58

6. Felten, J. E. \& Rees, M. J. 1972, A\&A, 17, 226

7. Sunyaev, R. A. \& Titarchuk, L. G. 1980, A\&A, 86, 121

8. Kompaneets, A. S. 1957, Sov. Phys. - JETP, 4, 730

9. Titarchuk, L. 1994, ApJ, 434, 570

10. Zdziarski, A. A., Johnson, W. N., \& Magdziarz, P. 1996, MNRAS, 283, 193

11. Coppi, P. S. 1992, MNRAS, 258, 657

12. Poutanen, J. \& Svensson, R. 1996, ApJ, 470, 249

13. Zdziarski, A. A., et al. 1990, ApJ, 363, L1

14. Coppi, P. S., Zdziarski, A. A., \& Madejski, G. M. 1998, in preparation

15. White, T. R., Lightman, A. P., \& Zdziarski, A. A. 1988, ApJ, 331, 939

16. Magdziarz, P. \& Zdziarski, A. A. 1995, MNRAS, 273, 837

17. Done, C., Mulchaey, J. S., Mushotzky, R. F., \& Arnaud, K. A. 1992, ApJ, 395, 275

18. Haardt, F., Maraschi, L., Ghisellini, G. 1994, ApJ, 432, L95

19. Stern, B. E., Poutanen, J., Svensson, R., Sikora, M., \& Begelman, M. C. 1995, ApJ, 449, L13

20. Svensson, R. 1996, in "Relativistic Astrophysics: A Conference in Honour of Professor I. D. Novikov's 60th Birthday", edited by B. J. T. Jones \& D. Markovic, 235

21. Svensson, R. 1996, ApJS, 120C, 475

22. Poutanen, J., Nagendra, K. N., \& Svensson, R. 1996, MNRAS, 283, 892

23. Fabian, A. C. et al. 1989, MNRAS, 238, 729

24. Gierliński, M., et al. 1997, in "Proc. 4th Compton Symposium", edited by C. D. Dermer, M. S. Strickman, \& J. D. Kurfess, in press

25. Zycki, P. T., Done, C., \& Smith, D. A. 1997, ApJ, 488, L113

26. Sunyaev, R. A. \& Trümper, J. 1979, Nature, 279, 506

27. Grebenev, S., et al. 1993, A\&AS, 97, 281

28. Svensson, R. 1994, ApJS, 92, 585

29. Nandra, K. \& Pounds, K. A. 1994, MNRAS, 268, 405

30. White, N. E., Fabian, A. C., \& Mushotzky, R. F. 1984, A\&A, 133, L9

31. Zdziarski, A. A., Johnson, W. N., Poutanen, J., Magdziarz, P., \& Gierliński, M. 1997, in "The Transparent Universe", edited by C. Winkler, T. J.-L. Courvoisier, \& Ph. Durouchoux, ESA SP-382, 373

32. Gondek, D., et al. 1996, MNRAS, 282, 646

33. Ebisawa, K., Ueda, Y., Inoue, H., Tanaka, Y., \& White N. E. 1996, ApJ, 467, 419

34. Gierliński, M., et al. 1997, MNRAS, 288, 958

35. Maisack, M. et al. 1995, A\&A, 298, 400

36. Johnson, W. N., et al. 1997, in "Proc. 4th Compton Symposium", in press 
37. McConnell, M. L., et al. 1994, ApJ, 424, 933

38. Ling, J. C., et al. 1997, ApJ, 484, 375

39. Van Dijk, R., et al. 1995, A\&A, 296, L33

40. Li, H., Kusunose, M., \& Liang, E. P. 1996, A\&AS, 120C, 167

41. Poutanen, J. \& Coppi, P. S. 1998, Physica Scripta, in press (astro-ph/9711316)

42. Liang, E. P. T. 1979, ApJ, 231, L111

43. Haardt, F. \& Maraschi, L. 1993, ApJ, 413, 507

44. Poutanen, J., Krolik, J. H., \& Ryde, F. 1997, MNRAS, 292, L21

45. Dove, J. B., Wilms, J., Maisack, M., \& Begelman M. C. 1997, ApJ, 487, 759

46. Zdziarski, A. A., Poutanen, J., Mikołaewska, J., Gierliński, M., Ebisawa, K., \& Johnson, W. N. 1998, MNRAS, submitted

47. Galeev, A. A., Rosner, R., \& Vaiana, G. S. 1979, ApJ, 229, 318

48. Nayakshin, S. \& Melia, F. 1998, ApJ, submitted (astro-ph/9709286)

49. Bisnovatyi-Kogan, G. S. \& Blinnikov, S. I. 1977, A\&A, 59, 111

50. Lightman, A. P. 1974, ApJ, 194, 429

51. Celotti, A., Fabian, A. C., \& Rees, M. J. 1992, MNRAS, 255, 419

52. Collin-Souffrin, S., Czerny, B., Dumont, A.-M., \& Życki, P. T. 1996, A\&A, 314, 393

53. Kuncic, Z., Celotti, A., \& Rees, M. J. 1997, MNRAS, 284, 717

54. Krolik, J. H., Madau, P., \& Życki, P. T. 1994, ApJ, 420, L57

55. Ghisellini, G., Haardt, F., \& Matt, G. 1994, MNRAS, 267, 743

56. Van der Klis, M. 1995, in "X-ray Binaries", Cambridge Astrophysics Series, vol. 26, edited by W. H. G. Lewin, J. van Paradijs, E. P. J. van den Heuvel, 252

57. Miyamoto, S., Kitamoto, S., Mitsuda, K., \& Dotani, T. 1991, Nature, 336, 450

58. Cui, W., Zhang, S. N., Focke, W., \& Swank, J. H. 1997, ApJ, 484, 383

59. Kazanas, D., Hua, X.-M., \& Titarchuk, L. 1997, ApJ, 480, 735

60. Hua, X.-M., Kazanas, D., \& Titarchuk, L. 1997, ApJ, 482, L57

61. Gierliński, M., et al. 1998, in preparation

62. Cui, W., et al. 1997, ApJ, 474, L57

63. Grove, J. E., et al. 1997, in "The Transparent Universe", edited by C. Winkler, T. J.-L. Courvoisier, \& Ph. Durouchoux, ESA SP-382, 197

64. Grove, J. E., et al. 1997, in "Proc. 4th Compton Symposium", in press

65. Grove, J. E., et al. 1998, ApJ, in press

66. Phlips, B. F., et al. 1996, ApJ, 465, 907

67. Iyudin, A. F., et al. 1998, in preparation

68. Ebisawa, K., Titarchuk, L., \& Chakrabarti, S. K. 1996, PASJ, 48, 59

69. Titarchuk, L., Mastichiadis, A., \& Kylafis, N. D. 1997, ApJ, 487, 834

70. Tanaka, Y. 1991, in "Iron line diagnostics in X-ray sources", edited by A. Treves, G. S. Perrola, L. Stella, Lecture Notes in Physics, vol. 385, 98

71. Li, H., Kusunose, M., \& Liang, E. P. 1996, ApJ, 460, L29

72. Liang, E. P. \& Narayan, R. 1997, in "Proc. 4th Compton Symposium", in press

73. Paciesas, W. S., et al. 1997, in "Proc. 4th Compton Symposium", in press

74. Kuznetsov, S., et al., 1997, MNRAS, 292, 651

75. Mineshige, S., Kusunose, M., \& Matsumoto, R. 1995, ApJ, 445, L43

76. Esin, A. A., McClintock, J. E., \& Narayan, R. 1997, ApJ, 489, 865 